\title{
Agreement Between the JCDCG, Revised NCEP-ATPIII, and IDF Definitions of Metabolic Syndrome in a Northwestern Chinese Population
}

\author{
Fei Sun · Bin Gao · Li Wang · Ying Xing · Jie Ming · Jie Zhou • \\ Jianfang $\mathrm{Fu} \cdot \mathrm{Xiaomiao} \mathrm{Li} \cdot$ Shaoyong Xu $\cdot$ Guocai Liu • \\ Qiuhe Ji (D)
}

Received: April 6, 2018 / Published online: May 28, 2018

(C) The Author(s) 2018

\begin{abstract}
Introduction: The Joint Committee for Developing Chinese Guidelines (JCDCG) introduced the Chinese definition for metabolic syndrome (MS), which has been verified in southern Chinese people but not in northwestern Chinese people. We evaluated the MS definition proposed by the JCDCG in a northwestern Chinese population, in comparison with those of the revised National Cholesterol Education Program Adult Treatment Panel III (NCEP-ATPIII) and the International Diabetes Federation (IDF).
\end{abstract}

Fei Sun and Bin Gao contributed equally to this work.

Enhanced digital features To view enhanced digital features for this article go to https://doi.org/10.6084/ m9.figshare.6264902.

Electronic supplementary material The online version of this article (https://doi.org/10.1007/s13300018-0446-9) contains supplementary material, which is available to authorized users.

F. Sun $\cdot$ B. Gao $\cdot$ L. Wang $\cdot$ Y. Xing $\cdot$ J. Ming

J. Zhou · J. Fu · X. Li · S. Xu · G. Liu · Q. Ji ( $₫)$

Department of Endocrinology, Xijing Hospital,

Fourth Military Medical University, Xi'an, Shaanxi,

China

e-mail: qiuheji@hotmail.com

G. Liu

The Third Department of Internal Medicine, The

273 Hospital of Chinese PLA, Korla, Xinjiang, China
Methods: This population-based cross-sectional study was a part of the China National Diabetes and Metabolic Disorders Study conducted in Shaanxi province. We included 3243 participants aged $\geq 20$ years. The age-adjusted MS prevalence was assessed per the 2007 Chinese population structure. The agreement between different definitions was assessed by the kappa statistic.

Results: The standardized prevalence of JCDCG-MS, revised ATPIII-MS, and IDF-MS was $22.4 \%, 29.4 \%$, and $24.9 \%$, respectively. Among women, the agreement of the JCDCG definition with the revised ATPIII and the IDF definition was not good $(\kappa=0.599$ and 0.601 , respectively); $54.6 \%$ of the revised ATPIII-MS and 56\% of the IDF-MS were defined as MS according to the JCDCG definition. Among men, the agreement of JCDCG definition with the revised ATPIII and IDF definitions was very good $(\kappa=0.863)$ and substantial $(\kappa=0.741)$, respectively.

Conclusion: The agreement of the JCDCG definition with the revised ATPIII and IDF definitions was insufficient in women. Compared with the other two definitions, the JCDCG definition underestimates MS prevalence in northwestern women.

Keywords: Agreement; Cardiovascular diseases; Chinese; Diabetes; Metabolic syndrome; Prevalence; Waist circumference 


\section{INTRODUCTION}

Metabolic syndrome (MS) has attracted increased attention due to its significant impact on cardiovascular diseases (CVD) and diabetes [1-3]. In the past few decades, different definitions of MS have been proposed by several organizations. For example, the World Health Organization (WHO) [4], the US National Cholesterol Education Program Adult Treatment Panel III (NCEP-ATPIII) [5], and International Diabetes Federation (IDF) [6] have put forward their interpretations. Before long, the American Heart Association/National Heart, Lung, and Blood Institute (AHA/NHLBI) revised the ATPIII definition [7]. However, there is no consensus on the definition of MS worldwide. Studies revealed that the impact of different definitions of MS on the risk of future CVD and diabetes is discrepant $[8,9]$.

In China, the Joint Committee for Developing Chinese Guidelines (JCDCG) suggested a Chinese definition for MS [10]. The JCDCG version has been applied as a useful tool to investigate MS and predict the risk of CVD in the Chinese population [11-14]. A communitybased cohort study in southeast China revealed that only MS defined by the JCDCG, not those proposed by IDF and ATPIII, was associated with an increased risk of CVD events in Chinese women [15]. However, in China, different regions have their own lifestyles, and differences are especially between the south and the north, which may influence the metabolic status [16-18]. Accordingly, some studies had a different conclusion that the JCDCG's definition was not appropriate for defining MS and predicting acute coronary syndrome among Chinese people $[19,20]$. Therefore, there is an urgent need to determine whether the JCDCG definition is more appropriate than other definitions used effectively in the world, especially in northwestern Chinese people.

In order to provide solid proof for the determination, we conducted a populationbased study by using data from the China National Diabetes and Metabolic Disorders Study (CNDMDS) in Shaanxi province, northwest China, and evaluated the JCDCG, revised
ATPIII (Definition proposed by AHA/NHLBI), and IDF for the diagnosis of MS. We also investigated the agreement between the JCDCG definition and the two aforementioned definitions that are widely used.

\section{METHODS}

\section{Participants}

This study was a subsection of the second stage of the CNDMDS, a representative cross-sectional cohort of Chinese adults from June 2007 to May 2008. The details of the CNDMDS can be found in a previous study [21]. In brief, a multistage, stratified sampling method was used to select a representative cohort aged $\geq 20$ years from the general population in Shaanxi province (more details can be found in our previous study) [22].

A total of 3930 individuals who had lived in their residence for $\geq 5$ years were randomly chosen and invited to participate in the study. Of them, 3298 individuals completed the survey and examination (overall response rate, 83.9\%). After excluding 55 individuals who had missing data for waist circumference (WC), fasting serum glucose levels, 2-h postprandial glucose levels, serum triglyceride levels, serum highdensity lipoprotein cholesterol (HDL-C) levels, or blood pressure (BP), we finally included 3243 subjects with complete data in the study.

\section{Compliance with Ethics Guidelines}

The Institutional Review Boards of Xijing Hospital, Fourth Military Medical University approved this study. Written informed consent was obtained from each participant prior to data collection.

\section{Measurements}

A standard questionnaire was administered by well-trained staff to obtain information including the demographic characteristics, lifestyle risk factors, personal medical history, family history of diseases, educational level, cigarette 
smoking, alcohol drinking, and physical activity. Height and body weight were measured in an upright position, to the nearest $0.5 \mathrm{~cm}$ and $0.1 \mathrm{~kg}$, respectively. The WC measurements were taken at the end of a normal exhalation and were measured to the nearest $0.1 \mathrm{~cm}$ from the midpoint between the lower borders of the rib cage and the anterior superior iliac spine. Body mass index (BMI) was calculated as weight in kilograms divided by the square of height in meters. The mean of two BP measurements was used. Readings of systolic BP and diastolic BP were taken $5 \mathrm{~min}$ apart in the resting state.

An oral glucose-tolerance test was performed for each subject. All participants were required to fast for at least $8 \mathrm{~h}$ beforehand, the participants without history of diabetes were subject to a 75-g oral glucose tolerance test, and those with a history of diabetes were given a bread meal test for safety reasons. Vein blood samples were drawn at 0,30 , and $120 \mathrm{~min}$ after glucose or carbohydrate load. The plasma glucose (PG) levels including fasting PG and $2 \mathrm{~h}$ PG (2hPG) were measured. Furthermore, high-density lipoprotein cholesterol (HDL-C), low-density lipoprotein cholesterol (LDL-C), total cholesterol (TC), and triglyceride (TG) levels in the serum were measured using commercially available reagents at the clinical biochemical laboratory, Xijing Hospital, Xi'an, China.

\section{Diagnosing Standard}

According to the JCDCG, MS is defined if there are more than three of the following abnormalities: (1) Central obesity (WC $>90 \mathrm{~cm}$ for men and $>85 \mathrm{~cm}$ for women); (2) Elevated triglyceride level ( $\geq 1.7 \mathrm{mmol} / \mathrm{L})$ or receipt of specific treatment for this lipid abnormality; (3) Reduced HDLC level $(<1.04 \mathrm{mmol} / \mathrm{l})$ or specific treatment for this lipid abnormality; (4) Elevated BP ( $\geq 130$ / $85 \mathrm{mmHg}$ or current treatment for hypertension) or previously diagnosed hypertension; (5) Elevated PG level (fasting PG $\geq 6.1 \mathrm{mmol} / \mathrm{L}$ or $2 \mathrm{~h}$ postprandial $\mathrm{PG} \geq 7.8 \mathrm{mmol} / \mathrm{L}$ ) or previously diagnosed diabetes mellitus [10].

The revised ATPIII definition was defined as three or more of the following abnormalities: (1) WC of $90 \mathrm{~cm}$ for men of Asian origin and
$80 \mathrm{~cm}$ for women of Asian origin; (2) Triglyceride level of $1.7 \mathrm{mmol} / \mathrm{L}$ or receipt of drug treatment for elevated triglyceride levels; (3) HDL-C level of $1.03 \mathrm{mmol} / \mathrm{L}$ in men and $1.29 \mathrm{mmol} / \mathrm{L}$ in women or receipt of drug treatment for reduced HDL-cholesterol level; (4) High blood pressure of 130/85 $\mathrm{mmHg}$ or receipt of drug treatment for hypertension; (5) Impaired fasting PG level of $5.6 \mathrm{mmol} / \mathrm{L}$ or receipt of drug treatment for elevated glucose level [7].

According to the IDF definition, MS is defined as central obesity (WC $\geq 90 \mathrm{~cm}$ for Chinese men and $\geq 80 \mathrm{~cm}$ for Chinese women) along with two or more of the following abnormalities: (1) Triglyceride level of $1.7 \mathrm{mmol} / \mathrm{L}$ or receipt of specific treatment for this lipid abnormality; (2) HDL-C level of $1.03 \mathrm{mmol} / \mathrm{L}$ in men and $1.29 \mathrm{mmol} / \mathrm{L}$ in women or receipt of specific treatment for this lipid abnormality; (3) BP of 130/85 mmHg or receipt of treatment of previously diagnosed hypertension; (4) Fasting PG level of $5.6 \mathrm{mmol} /$ L or previously diagnosed type 2 diabetes [6].

\section{Statistical Analysis}

Continuous variables were expressed as the mean \pm standard deviation, and categorical variables are presented as proportions. $P$ values $<0.05$ were considered statistically significant. To compare continuous variables between groups, a $t$ test was used. To compare categorical variables between groups, the chi-square test was used. Linear trends for sex- and age-specific mean or proportion were tested using analysis of variance linear test (polynomial) or chisquare test for linear-by-linear association, respectively. A binary logistic regression analysis was adopted to compare the prevalence of MS between genders after adjusting for age. Agreement between different MS definitions was evaluated by the $\kappa$ value (poor, $\kappa \leq 0.20$; fair, $\quad \kappa=0.21-0.40 ;$ moderate, $\kappa=0.41-0.60$; substantial, $\kappa=0.61-0.80$; very good, $\kappa>0.80$ ) [23]. As our study was conducted in 2007-2008, the percentage values of MS prevalence were standardized by the direct method according to the Chinese population structure in 2007 [24]. 
A database was established using EpiData 3.1 software. Statistical analyses were carried out using the International Business Machines Corporation Statistical Package of Social Science for Windows version 22.0 (IBM Corp., Armonk, NY, USA).

\section{RESULTS}

\section{Characteristics of Study Population}

The baseline characteristics of the study subjects are shown in Table 1. This study included 3243 individuals (1359 men, 1884 women; men/women $0.721 ;$ age of men $44.3 \pm 14.0$ years, women $44.1 \pm 13.7$ years), with a mean BMI of $23.9 \mathrm{~kg} / \mathrm{m}^{2}$. Men had a significantly higher BMI, WC, triglyceride level, LDL-C level, education level, and proportion of alcohol drinking and cigarette smoking, whereas women had significantly higher HDL-C levels. The mean level of HDL-C was higher in women than in men. The systolic BP, diastolic BP, TC, fasting PG, 2hPG, and proportion of subjects who performed physical activity showed no significant differences between the two genders.

Table 1 Characteristics of the study population

\begin{tabular}{llllr}
\hline Variables & Total $(\boldsymbol{n}=\mathbf{3 2 4 3})$ & Men $(\boldsymbol{n}=\mathbf{1 3 5 9})$ & Women $(\boldsymbol{n}=\mathbf{1 8 8 4})$ & $\boldsymbol{P}$ value \\
\hline Age $($ years $)$ & $44.2 \pm 13.8$ & $44.3 \pm 14.0$ & $44.1 \pm 13.7$ & 0.615 \\
Educational level $(n \%)$ & & & \\
Elementary school or below & $730(22.5)$ & $209(15.4)$ & $521(27.7)$ & $<0.001$ \\
Middle school & $1601(49.4)$ & $710(52.2)$ & $891(47.3)$ & 0.007 \\
Collage or above & $912(28.1)$ & $442(32.5)$ & $470(24.9)$ & $<0.001$ \\
Physical activity $(n \%)$ & $1224(37.7)$ & $534(39.3)$ & $690(36.6)$ & 0.139 \\
Alcohol drinking $(n \%)$ & $801(24.7)$ & $694(51.1)$ & $107(5.7)$ & $<0.001$ \\
Cigarette smoking $(n \%)$ & $770(23.7)$ & $745(54.8)$ & $25(1.3)$ & $<0.001$ \\
BMI $\left(\mathrm{kg} / \mathrm{m}^{2}\right)$ & $23.9 \pm 1.40$ & $24.3 \pm 4.37$ & $23.6 \pm 3.88$ & $<0.001$ \\
WC $(\mathrm{cm})$ & $81.9 \pm 10.2$ & $85.8 \pm 9.72$ & $79.1 \pm 9.50$ & $<0.001$ \\
SBP $(\mathrm{mmHg})$ & $120.9 \pm 20.2$ & $121.3 \pm 20.7$ & $120.5 \pm 19.9$ & 0.241 \\
DBP $(\mathrm{mmHg})$ & $76.7 \pm 11.6$ & $76.6 \pm 11.8$ & $76.7 \pm 11.5$ & 0.836 \\
TC $(\mathrm{mmol} / \mathrm{L})$ & $4.71 \pm 1.03$ & $4.72 \pm 1.02$ & $4.70 \pm 1.03$ & 0.570 \\
TG $(\mathrm{mmol} / \mathrm{L})$ & $1.56 \pm 1.28$ & $1.75 \pm 1.50$ & $1.43 \pm 1.07$ & $<0.001$ \\
HDL-C $(\mathrm{mmol} / \mathrm{L})$ & $1.29 \pm 0.31$ & $1.21 \pm 0.28$ & $1.35 \pm 0.31$ & $<0.001$ \\
LDL-C $(\mathrm{mmol} / \mathrm{L})$ & $2.64 \pm 0.80$ & $2.68 \pm 0.76$ & $2.61 \pm 0.82$ & 0.020 \\
FPG $(\mathrm{mmol} / \mathrm{L})$ & $5.23 \pm 1.40$ & $5.25 \pm 1.43$ & $5.21 \pm 1.39$ & 0.288 \\
2hPG $(\mathrm{mmol} / \mathrm{L})$ & $6.52 \pm 3.29$ & $6.44 \pm 3.23$ & $6.58 \pm 3.32$ & 0.244 \\
\hline$P$ F & & & &
\end{tabular}

$P$ values are from $t$ tests between genders

$B M I$ body mass index, $W C$ waist circumference, $S B P$ systolic blood pressure, $D B P$ diastolic blood pressure, $T C$ total cholesterol, $T G$ triglycerides, $H D L$ - $C$ high-density lipoprotein cholesterol, $L D L-C$ low-density lipoprotein cholesterol, $F P G$ fasting plasma glucose, $2 h P G$ 2-h plasma glucose 


\section{Standardized Prevalence of MS}

The standardized MS prevalence rates of the study population as per the JCDCG-MS, revised ATPIII-MS, and IDF-MS were $22.4 \%, 29.4 \%$, and $24.9 \%$, respectively (Table 2 ). The prevalence rates of JCDCG-MS, revised ATPIII-MS, and IDFMS were $27.0 \%, 27.9 \%$, and $22.7 \%$ in men and
$18.0 \%, 30.8 \%$, and $27.0 \%$ in women, respectively. As compared to men, more women had MS according to the IDF definition $(27.9 \%$ vs. $30.8 \%, P=0.012$ ) and revised ATPIII definition (22.7\% vs. $27.0 \%, P<0.001)$. In contrast, more men than women were diagnosed with MS as per the JCDCG definition $(27.0 \%$ vs. $18.0 \%$, $P<0.001)$. Linear trends in the prevalence of

Table 2 Standardized proportion (95\% confidence interval) of metabolic syndrome using the JCDCG, revised ATPIII, and IDF definition

\begin{tabular}{|c|c|c|c|}
\hline & JCDCG-MS & Revised ATPIII-MS & IDF-MS \\
\hline Overall & $22.4(21.0-23.8)^{a}$ & $29.4(27.8-31.0)^{\mathrm{a}}$ & $24.9(23.4-26.4)^{\mathrm{a}}$ \\
\hline Men & $27.0(24.6-29.4)^{b}$ & $27.9(25.5-30.3)^{\mathrm{b}}$ & $22.7(20.5-24.9)^{\mathrm{b}}$ \\
\hline Women & $18.0(16.3-19.7)^{\mathrm{b}}$ & $30.8(28.7-32.9)^{\mathrm{b}}$ & $27.0(25.0-29.0)^{\mathrm{b}}$ \\
\hline$P^{c}$ & $<0.001$ & 0.012 & $<0.001$ \\
\hline \multicolumn{4}{|c|}{ Men age (years) } \\
\hline $20-29$ & $12.9(8.6-17.2)$ & $12.4(8.2-16.6)$ & $10.3(6.4-14.2)$ \\
\hline $30-39$ & $28.5(23.6-33.4)$ & $31.5(26.5-36.6)$ & $27.0(22.2-31.8)$ \\
\hline $40-49$ & $33.8(28.5-39.1)$ & $35.2(29.9-40.5)$ & $28.4(23.4-33.4)$ \\
\hline $50-59$ & $39.4(33.6-45.2)$ & $38.5(32.7-44.3)$ & $26.6(21.3-31.9)$ \\
\hline $60-69$ & $42.6(34.7-50.5)$ & $40.6(32.7-48.5)$ & $32.6(25.1-40.1)$ \\
\hline$\geq 70$ & $26.7(16.5-37.0)$ & $26.7(16.5-37.0)$ & $24.2(14.3-34.1)$ \\
\hline$P^{\mathrm{d}}$ & $<0.001$ & $<0.001$ & $<0.001$ \\
\hline \multicolumn{4}{|c|}{ Women age (years) } \\
\hline $20-29$ & $3.2(1.2-5.2)$ & $8.7(5.6-11.9)$ & $7.7(4.7-10.7)$ \\
\hline $30-39$ & $6.7(4.4-9.0)$ & $16.7(13.3-21.2)$ & $13.8(10.6-17.0)$ \\
\hline $40-49$ & $17.8(14.4-21.2)$ & $37.1(32.8-41.5)$ & $29.9(25.8-34.0)$ \\
\hline $50-59$ & $34.8(30.0-39.6)$ & $54.1(49.1-59.1)$ & $49.2(44.2-54.2)$ \\
\hline $60-69$ & $40.4(33.5-47.3)$ & $57.9(51.0-64.8)$ & $49.8(42.8-56.8)$ \\
\hline$\geq 70$ & $43.1(32.1-54.1)$ & $49.3(38.2-60.4)$ & $49.3(38.2-60.4)$ \\
\hline$P^{\mathrm{d}}$ & $<0.001$ & $<0.001$ & $<0.001$ \\
\hline
\end{tabular}

JCDCG Joint Committee for Developing Chinese Guidelines, ATPIII Adult Treatment Panel III, IDF International Diabetes Federation

${ }^{a}$ Age- and sex-adjusted percentages

b Age-adjusted percentages for men or women. The percentage values were standardized by the direct method according to the Chinese population structure in 2007

${ }^{c}$ Adjusted for age using binary logistic analysis

d $P$ value for linear trend from the chi-square test for linear-by-linear association 
MS showed a significant increase with age in both genders according to all three definitions (Table 2).

\section{Crude Prevalence of Metabolic Abnormalities According to JCDCG, Revised ATPIII, and IDF Criteria}

In men, except for the elevated PG and central obesity, the prevalence of the other metabolic abnormalities according to the revised ATPIII (IDF) criteria was similar in either MS group or non-MS group. About 74.0\% of JCDCG-MS and $81 \%$ of ATPIII-MS were defined as MS by the IDF definition, $91.0 \%$ of JCDCG-MS and all the IDFMS were defined as MS by the revised ATPIII definition, and $87.9 \%$ of ATPIII-MS and $88.3 \%$ of IDF-MS were defined as MS by the JCDCG definition (Fig. 1).

In women, according to JCDCG criteria, the crude prevalence of elevated TG, elevated BP, elevated PG, central obesity, and reduced HDL$\mathrm{C}$ was higher in JCDCG-MS subjects. In addition, the prevalence rates of the metabolic abnormalities mentioned above were not lower in non-MS subjects defined by the JCDCG definition than the non-MS subjects defined by the other two definitions. According to the JCDCG definition, $54.6 \%$ of ATPIII-MS and $56.0 \%$ of IDF-MS were defined as MS; $14.4 \%$ and $17.0 \%$ of non-MS subjects according to the JCDCG definition were defined as MS by the IDF definition and revised ATPIII definition, respectively (Fig. 2).

\section{Agreement Between JCDCG, Revised ATPIII, and IDF Criteria for MS Diagnosis}

To verify the agreement between the different definitions of MS for both genders, we used the $\kappa$ statistic. A substantial overall agreement $(\kappa=0.712)$ was observed between the JCDCG and revised ATPIII definitions, and a moderate overall agreement $(\kappa=0.648)$ was observed between the JCDCG and IDF definitions (Table 3$)$. When analyzed by gender, very good agreement $(\kappa=0.863)$ was observed between the JCDCG and revised ATPIII definitions for men, but only a moderate agreement $(\kappa=0.599)$ was observed for women. A substantial agreement was found for both genders between the JCDCG and IDF definitions (overall, $\quad \kappa=0.648 ; \quad$ men, $\quad \kappa=0.741$; women, $\kappa=0.601)$.
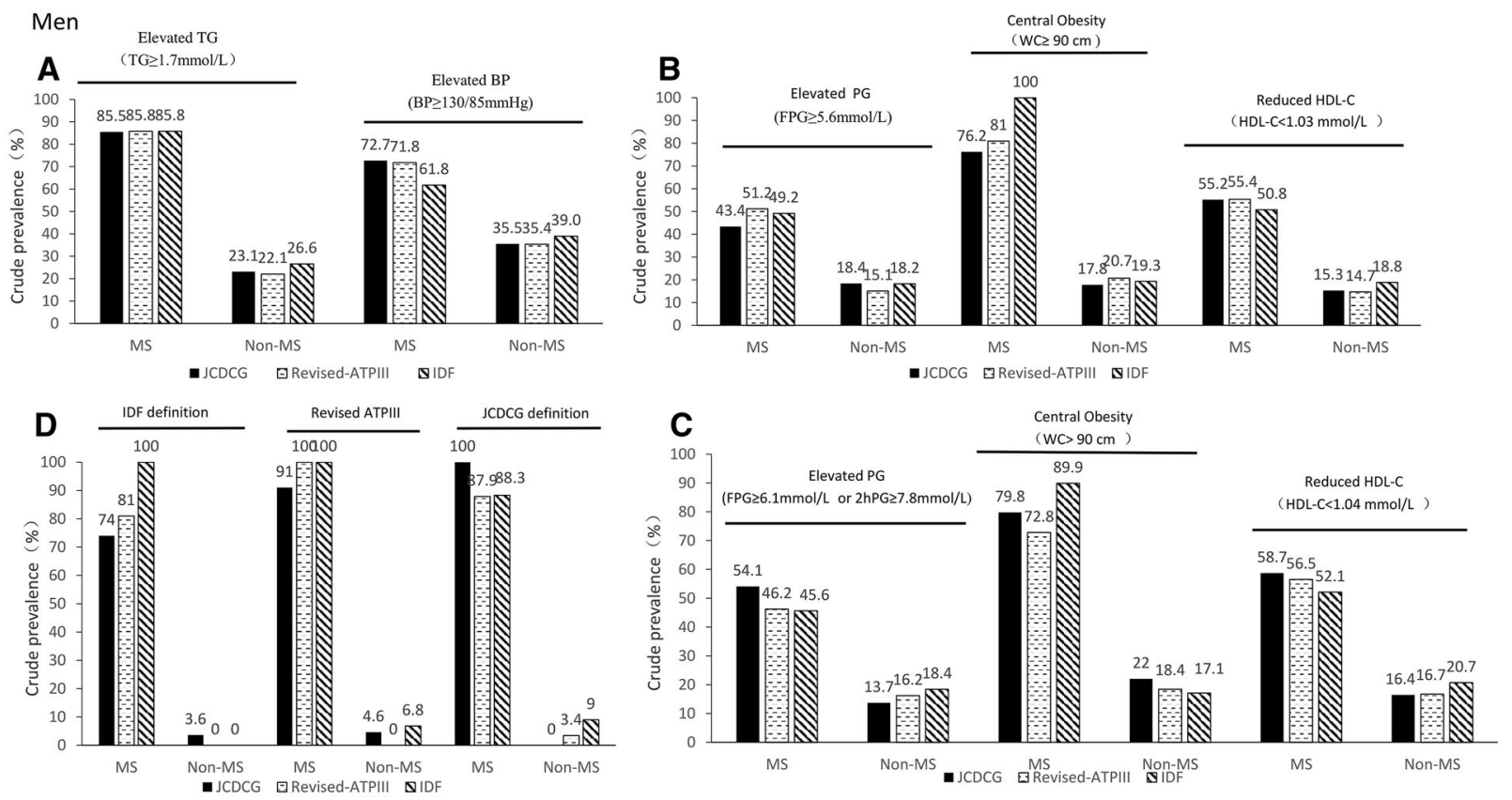

Fig. 1 Crude prevalence of metabolic abnormalities according to JCDCG, revised ATPIII, and IDF criteria in men 

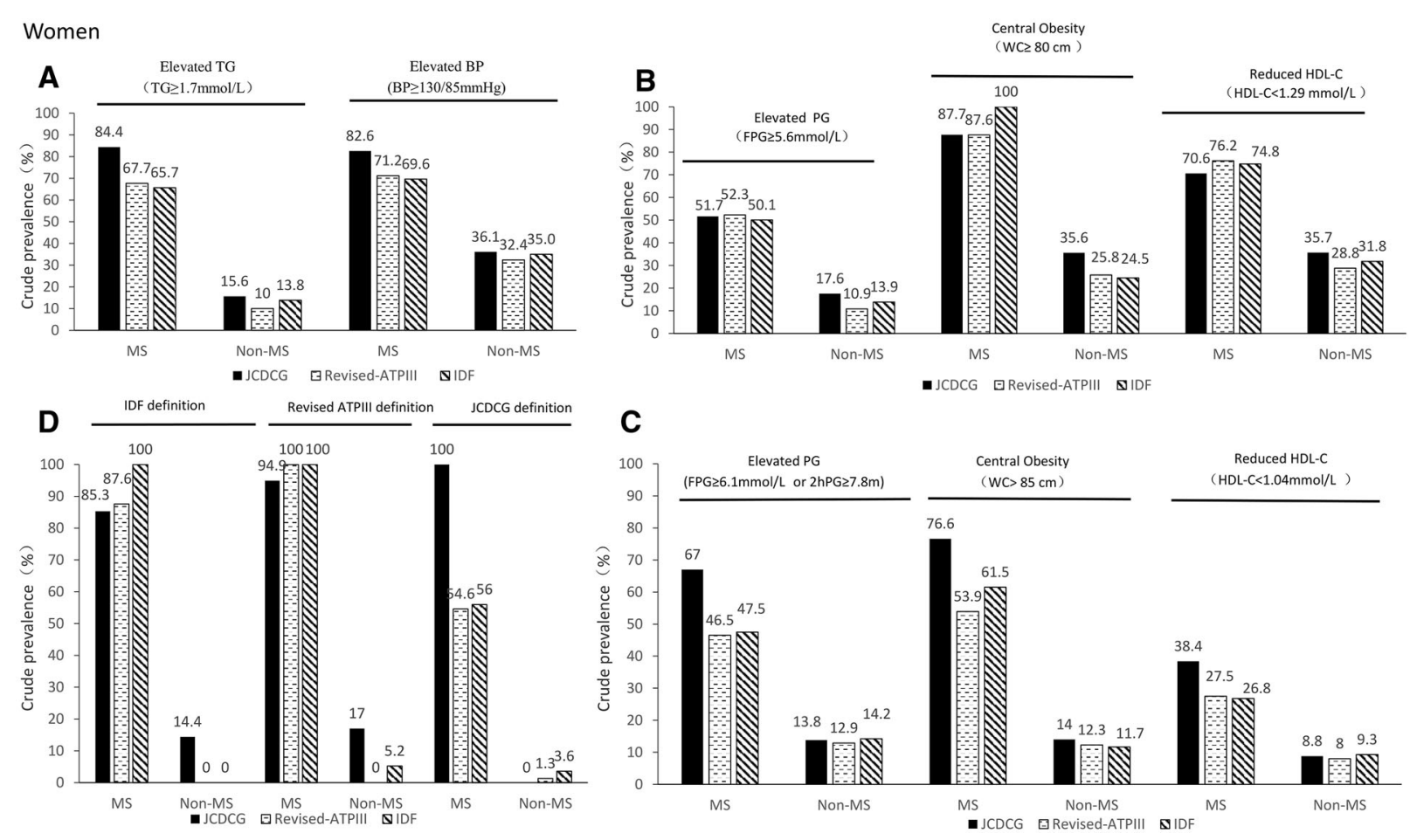

Fig. 2 Crude prevalence of metabolic abnormalities according to JCDCG, revised ATPIII, and IDF criteria in women

\section{DISCUSSION}

We conducted a population-based study in Shaanxi province, northwest China, to compare the JCDCG definition of MS with the IDF and the revised ATPIII definitions. We found that the agreement between the JCDCG definition and the revised ATPIII or the IDF definitions was not good enough, especially in northwestern Chinese women. JCDCG only recognized $56.0 \%$ of IDF-MS and $54.6 \%$ of revised ATP-MS; also, there were still $14.4 \%$ of IDF-MS and $17.0 \%$ of revised ATP-MS defined as non-MS according to JCDCG. These results indicated that the JCDCG definition may underestimate the prevalence of MS in women compared with the other two definitions. Hence, we should consider the gender difference and further verify the reliability of the JCDCG definition in assessing MS in northwestern Chinese women.

Our results showed that $22.4 \%$ of Shaanxi adults met the MS criteria according to the JCDCG definition, which is slightly higher than the national level. In a nationwide study performed in 2007-2008, which enrolled 46,024 participants, the prevalence of MS according to the JCDCG definition was $21.9 \%$ [13]. Varied prevalence was observed in different regions of China. A community-based study conducted in Shanghai, southeast China, revealed that $24.4 \%$ of study subjects met the MS definition of JCDCG. The varied prevalence of MS between northern and southern areas of China may be partially explained by different lifestyles, e.g., physical exercise, education level, and economic status $[16,25]$. In addition to these factors, studies have also revealed that the MS prevalence depends on the definition used [26-28]. The community-based study in Shanghai also revealed moderate to good agreement between the JCDCG definition with the IDF and ATPIII definition, with kappa value between 0.635 and 0.825 in both women and men. In spite of all these studies, there was lack of data evaluating JCDCG compared with other definitions in northern Chinese populations. As we acknowledged, we first revealed the agreement of the JCDCG definition with other definitions in northwestern Chinese people. 
Table 3 Agreement of the JCDCG with the revised ATPIII and IDF criteria for the diagnosis of MS

\begin{tabular}{|c|c|c|c|c|}
\hline & \multicolumn{3}{|c|}{ JCDCG } & \multirow[t]{2}{*}{$K$ value } \\
\hline & + & - & Total & \\
\hline \multicolumn{5}{|c|}{ Revised ATPIII } \\
\hline \multicolumn{5}{|l|}{ Overall } \\
\hline+ & 748 & 330 & 1078 & \multirow[t]{3}{*}{0.712} \\
\hline- & 59 & 2106 & 2165 & \\
\hline Total & 807 & 2436 & 3243 & \\
\hline \multicolumn{5}{|l|}{ Men } \\
\hline+ & 386 & 43 & 429 & \multirow[t]{2}{*}{0.863} \\
\hline- & 38 & 892 & 930 & \\
\hline Total & 424 & 935 & 1359 & \\
\hline \multicolumn{5}{|c|}{ Women } \\
\hline+ & 362 & 287 & 649 & \multirow[t]{3}{*}{0.599} \\
\hline- & 21 & 1214 & 1235 & \\
\hline Total & 383 & 1501 & 1884 & \\
\hline \multicolumn{5}{|l|}{ IDF } \\
\hline \multicolumn{5}{|l|}{ Overall } \\
\hline+ & 635 & 273 & 908 & \multirow[t]{3}{*}{0.648} \\
\hline- & 171 & 2164 & 2335 & \\
\hline Total & 806 & 2437 & 3243 & \\
\hline \multicolumn{5}{|l|}{ Men } \\
\hline+ & 312 & 111 & 423 & \multirow[t]{3}{*}{0.741} \\
\hline- & 32 & 904 & 936 & \\
\hline Total & 344 & 1015 & 1359 & \\
\hline \multicolumn{5}{|l|}{ Women } \\
\hline+ & 240 & 23 & 263 & \multirow[t]{3}{*}{0.601} \\
\hline- & 180 & 976 & 1156 & \\
\hline Total & 420 & 999 & 1419 & \\
\hline
\end{tabular}

Agreement between the different MS definitions was evaluated by the $\kappa$ statistic (poor, $\kappa \leq 0.20$; fair, $\kappa=0.21-0.40$; moderate, $\kappa=0.41-0.60$; substantial, $\kappa=0.61-0.80$; very good, $\kappa>0.80$ )

JCDCG Joint Committee for Developing Chinese Guidelines, ATPIII Adult Treatment Panel III, IDF International Diabetes Federation 
In the current study, the agreement between the JCDCG definition and the other two was not very good in women. As we observed JCDCG only recognized $56.0 \%$ of IDF-MS and $54.6 \%$ of revised ATP-MS; also, there were still $14.4 \%$ of IDF-MS and $17.0 \%$ of revised ATP-MS defined as non-MS according to JCDCG. Furthermore, according to JCDCG criteria of the metabolic abnormalities, the crude prevalence of the five metabolic abnormalities was higher in both MS and non-MS subjects by JCDCG than the subjects defined by revised ATPIII and IDF definitions. These results all proved the poor agreement between JCDCG and the other two definitions in northwestern Chinese women. Comparison of the three criteria of MS shows that the different cutoffs of central obesity (waist circumference, 80 vs $85 \mathrm{~cm}$ ), reduced HDL-C (1.04 vs. $1.29 \mathrm{mmol} / \mathrm{l})$, and hyperglycemia (FPG $5.6 \mathrm{mmol} / \mathrm{l}$ vs. FPG $6.1 \mathrm{mmol} / \mathrm{l}$, 2hPG $7.8 \mathrm{mmol} / \mathrm{l}$ ) may lead to the different prevalence. Among those above, the most important criteria of metabolic syndrome should be based upon the visceral adiposity which controls the micro-inflammation, adiponectin, and adipocytokines that induce insulin resistance. The waist circumference of $85 \mathrm{~cm}$ for women was derived from visceral fat area (VFA) measured by magnetic resonance imaging in a southeastern Chinese population in Shanghai. Similarly, the cutoff point of waist circumference was also $85 \mathrm{~cm}$ in Japanese women, and it was determined by the visceral level area obtained by CT scan [29]. Recently an intervention trial based on a large population confirmed the validity of this criterion [30]. But it remains unclear whether the waist circumference of $85 \mathrm{~cm}$ for women is adapted to northwestern Chinese women; a prospective study should be conducted to verify this. Previous study has revealed that agreement between the JCDCG definition and other definitions (the WHO, IDF, and ATPIII definitions) was moderate to good in southeastern Chinese women [15]. With such different results in different regions, we should be cautious when using the JCDCG definition, and the JCDCG definition need to be verified by prospective study in northwestern Chinese women. So far, no study has observed such differences according to JCDCG and other widely used definitions in northwestern Chinese women.

In men, the agreement between JCDCG, IDF, and revised ATPIII definition was good. The prevalence of MS defined by IDF was slightly lower than the prevalence of MS defined by JCDCG and revised ATPIII definitions, possibly because central obesity has been regarded as a requisite criterion of the IDF definition. However, the prevalence of MS defined by JCDCG and revised ATPIII was close (JCDCG-MS: 27.0\%, revised ATPIII-MS: $27.9 \%$ ), because the only difference between the two definition is the cutoff of elevated PG. The prevalence rates of JCDCG-MS we observed in our present study were similar to the nationwide study $(27.0 \%$ vs. $25.8 \%$ ) and the community-based study in Shanghai $(27.0 \%$ vs. $26.1 \%)$ which was mentioned above [13, 15]. Based on the results above, the agreement between JCDCG and the other two definitions was good in men. It was very different from what we had observed in women for these three definitions.

Despite our important findings, our study has some limitations. First, as it was a crosssectional study, future prospective studies should be conducted to examine which definition of MS has better predictive power for the risk of CVD and diabetes. Second, as the study was conducted in northwest China, the conclusions from this study may not be generalizable to other areas. Finally, we cannot exclude the possibility of selection bias. For example, the prevalence of MS decreased in the group of subjects aged 70 or older. It may because there were few subjects in this group. There are also strengths in our study: our present study not only observed the prevalence of MS according to JCDCG but also compared JCDCG with the IDF and the revised ATPIII definitions in the northwestern Chinese population. In addition, our study assessed the definitions of MS in men and women separately.

\section{CONCLUSIONS}

The agreement of the JCDCG definition with the revised ATPIII and the IDF definitions was insufficient in women. The JCDCG definition 
may underestimate MS in the northwestern female population when compared with the other two definitions, most likely because of the loose cutoffs of central obesity in women. Therefore, the cutoffs of central obesity in northwestern Chinese women need to be verified, and we should be aware of the difference between the JCDCG definition and the other MS definitions when evaluating MS in northwestern Chinese women. Prospective studies may be needed to prove the capability of the definitions for predicting future cardiovascular disease and diabetes.

\section{ACKNOWLEDGEMENTS}

We thank all of the physicians and participants of the study for their co-operation and generous participation.

Funding. This study was supported by grants from Shaanxi Natural Science Foundation of China (2014JM2-8198), the National Natural Science Foundation of China (81300696), and the National Key R\&D Program of China (2017YFC1309803). The grants also funded the journal's article processing charges.

Authorship. All named authors meet the International Committee of Medical Journal Editors (ICMJE) criteria for authorship for this article, take responsibility for the integrity of the work as a whole, and have given their approval for this version to be published.

Authorship Contributions. All the authors have made a significant contribution to this manuscript. Fei Sun and Bin Gao contributed equally to this work. Qiuhe Ji was responsible for the study design. Fei Sun, Bin Gao, and Li Wang participated in the design of the study and drafted the manuscript. Ying Xing, Shaoyong $\mathrm{Xu}$, Jie Ming, Jie Zhou, Jianfang Fu, Xiaomiao Li, and Guocai Liu conducted the study and participated in the design and interpretation of the results or made important intellectual contributions to the interpretation of data or being involved in data collection. All authors are in agreement with the content of the manuscript.

Disclosures. Qiuhe Ji has attended advisory boards and received travel support, and been a speaker of Eli Lilly, Novo Nordisk, Merck \& Co., Merck Sharp \& Dohme China, Sanofi Aventis, Huadong Pharmaceuticals Company, and Medtronic, and received research grants from Novo Nordisk, Merck Sharp \& Dohme China, and AstraZeneca. The remaining authors (Fei Sun, Bin Gao, Li Wang, Ying Xing, Jie Ming, Jie Zhou, Jianfang Fu, Xiaomiao Li, Shaoyong Xu, Guocai Liu) have nothing to disclose.

Compliance with Ethics Guidelines. The Institutional Review Boards of Xijing Hospital, Fourth Military Medical University approved this study. Written informed consent was obtained from each participant prior to data collection.

Data Availability. The data sets generated and/or analyzed during the current study are available from the corresponding author on reasonable request.

Open Access. This article is distributed under the terms of the Creative Commons Attribution-NonCommercial 4.0 International License (http://creativecommons.org/licenses/ by-nc/4.0/), which permits any noncommercial use, distribution, and reproduction in any medium, provided you give appropriate credit to the original author(s) and the source, provide a link to the Creative Commons license, and indicate if changes were made.

\section{REFERENCES}

1. Alberti KG, Eckel RH, Grundy SM, et al. Harmonizing the metabolic syndrome: a joint interim statement of the International Diabetes Federation Task Force on Epidemiology and Prevention; National Heart, Lung, and Blood Institute; American Heart Association; World Heart Federation; International Atherosclerosis Society; and International Association for the Study of Obesity. Circulation. 2009;120:1640-5. 
2. Ford ES. Risks for all-cause mortality, cardiovascular disease, and diabetes associated with the metabolic syndrome: a summary of the evidence. Diabetes Care. 2005;28:1769-78.

3. Shin JA, Lee JH, Lim SY, et al. Metabolic syndrome as a predictor of type 2 diabetes, and its clinical interpretations and usefulness. J Diabetes Investig. 2013;4:334-43.

4. WHO. Definition, diagnosis and classification of diabetes mellitus and its complications. Geneva: World Health Organization; 1999.

5. National Cholesterol Education Program (NCEP) Expert Panel on Detection, Evaluation, and Treatment of High Blood Cholesterol in Adults (Adult Treatment Panel III). Third Report of The National Cholesterol Education Program (NCEP) Expert Panel on Detection, Evaluation, and Treatment of High Blood Cholesterol in Adults (Adult Treatment Panel III). JAMA. 2001;285:2486-97.

6. Alberti KG, Zimmet P, Shaw J. The metabolic syndrome-a new worldwide definition. Lancet. 2005;366:1059-62.

7. Grundy SM, Cleeman JI, Daniels SR, et al. Diagnosis and management of the metabolic syndrome: an American Heart Association/National Heart, Lung, and Blood Institute Scientific Statement. Circulation. $2005 ; 112: 2735-52$.

8. Khosravi-Boroujeni H, Ahmed F, Sadeghi M, et al. Does the impact of metabolic syndrome on cardiovascular events vary by using different definitions? BMC Public Health. 2015;15:1313.

9. Kastorini CM, Panagiotakos DB, Georgousopoulou $\mathrm{EN}$, et al. Metabolic syndrome and 10-year cardiovascular disease incidence: the ATTICA study. Nutr Metab Cardiovasc Dis. 2016;26:223-31.

10. Joint Committee for Developing Chinese Guidelines. Chinese guidelines on prevention and treatment of dyslipidemia in adults. Zhonghua Xin Xue Guan Bing Za Zhi. 2007;2007(35):390-419.

11. Hou XH, Zhu YX, Lu HJ, et al. Non-alcoholic fatty liver disease's prevalence and impact on alanine aminotransferase associated with metabolic syndrome in the Chinese. J Gastroenterol Hepatol. 2011;26:722-30.

12. Zhu Y, Zhang M, Hou X, et al. Cigarette smoking increases risk for incident metabolic syndrome in Chinese men-Shanghai diabetes study. Biomed Environ Sci. 2011;24:475-82.

13. Hou X, Lu J, Weng J, et al. Impact of waist circumference and body mass index on risk of cardiometabolic disorder and cardiovascular disease in
Chinese adults: a national diabetes and metabolic disorders survey. PLoS One. 2013;8:e57319.

14. Yu H, Zhang L, Bao Y, et al. Metabolic syndrome after Roux-en-Y gastric bypass surgery in Chinese obese patients with type 2 diabetes. Obes Surg. 2016;26:2190-7.

15. Wang C, Hou X, Bao Y, et al. The metabolic syndrome increased risk of cardiovascular events in Chinese-a community based study. Int J Cardiol. 2010;139:159-65.

16. Xu S, Ming J, Xing Y, et al. Regional differences in diabetes prevalence and awareness between coastal and interior provinces in China: a population-based cross-sectional study. BMC Public Health. 2013;13:299.

17. Pu Y, Liu L, Wang Y, et al. Geographic and sex difference in the distribution of intracranial atherosclerosis in China. Stroke. 2013;44:2109-14.

18. Zhao D, Wu N, Yang J, et al. The prevalence and associated risk factors of impaired glucose regulation in Chinese adults: a population-based crosssectional study. Int J Endocrinol. 2015;2015:731583.

19. Wang Q, Chair SY, Wong EM, et al. Comparing the predictive abilities of different metabolic syndrome definitions for acute coronary syndrome: a case-control study in Chinese adults. Metab Syndr Relat Disord. 2014;12:390-6.

20. Hu Z, Zhang R, Huang L, et al. A comparison of applicative effect with different diagnostic criteria of metabolic syndrome among the urban adult population. Zhonghua Yu Fang Yi Xue Za Zhi. 2014;48:507-11 (Chinese).

21. Yang W, Lu J, Weng J, et al. Prevalence of diabetes among men and women in China. N Engl J Med. 2010;362:1090-101.

22. Xu S, Ming J, Yang C, et al. Urban, semi-urban and rural difference in the prevalence of metabolic syndrome in Shaanxi province, northwestern China: a population-based survey. BMC Public Health. 2014;14:104.

23. Dg A. Practical statistics for medical research. London: Chapman and Hall; 1991.

24. National Bureau of Statistics of China. China statistical yearbook-2007. Beijing: China Statistics Press.http://www.stats.gov.cn/tjsj/ndsj/2007/ indexeh.htm.

25. Dalleck LC, Van Guilder GP, Quinn EM, et al. Primary prevention of metabolic syndrome in the 
community using an evidence-based exercise program. Prev Med. 2013;57:392-5.

26. Herrera-Enriquez K, Narvaez-Guerra O. Discordance of metabolic syndrome and abdominal obesity prevalence according to different criteria in Andean highlanders: a community-based study. Diabetes Metab Syndr. 2017;1:S359-64.

27. Maksimovic MZ, Vlajinac HD, Radak DJ, et al. Prevalence of the metabolic syndrome in patients with carotid disease according to NHLBI/AHA and IDF criteria: a cross-sectional study. BMC Cardiovasc Disord. 2012;12:2.
28. Foss-Freitas MC, Gomes PM, Andrade RC, et al. Prevalence of the metabolic syndrome using two proposed definitions in a Japanese-Brazilians community. Diabetol Metab Syndr. 2012;4:38.

29. Zimmet P, Magliano D, Matsuzawa Y, et al. The metabolic syndrome: a global public health problem and a new definition. J Atheroscler Thromb. 2005;12:295-300.

30. Tsushita K, Sh A, Miura K, et al. Rationale and descriptive analysis of specific health guidance: the nationwide lifestyle intervention program targeting metabolic syndrome in Japan. J Atheroscler Thromb. 2018;25:308-22. 